\title{
Cytokine levels in abdominal exudate predict prolonged postoperative ileus following surgery for colorectal carcinoma
}

\author{
PENGCHENG ZHU*, HONGHUA JIANG ${ }^{*}$, JIHONG FU, WEI CHEN, ZHONGCHUAN WANG and LONG CUI \\ Department of Colorectal Surgery, Xinhua Hospital Affiliated to Shanghai Jiaotong University School of Medicine, \\ Shanghai 200092, P.R. China
}

Received January 21, 2013; Accepted June 7, 2013

DOI: $10.3892 / 01.2013 .1465$

\begin{abstract}
The aim of the present study was to investigate whether the measurement of cytokines from abdominal exudate is valuable for the early diagnosis of prolonged postoperative ileus (PPOI) following colorectal surgery. In the present study, 100 consecutive patients who were scheduled to undergo elective resection for carcinoma of the sigmoid or rectum were investigated. Abdominal exudate was obtained via a drain tube following surgery for the detection of interleukin (IL)-1 $\beta$, IL-6 and tumour necrosis factor (TNF)- $\alpha$. The correlation among the cytokine levels on postoperative days 1 , 3 and 5 and the development of PPOI was investigated. Eight patients developed PPOI which was diagnosed 10-15 days postoperatively. No significant differences were observed among the peritoneal cytokine levels on postoperative days 1 and 3 in the 8 patients who developed PPOI when compared with those of the 92 patients that did not develop PPOI. By contrast, cytokine levels on postoperative day 5 were significantly higher in patients who developed PPOI when compared with those of patients that did not develop PPOI. The cytokine levels significantly increased during the first 5 days postoperatively in patients who developed PPOI and significantly decreased in patients who did not develop PPOI. The results of the present study showed that the increase in peritoneal IL-1 $\beta$, IL- 6 and TNF- $\alpha$ levels may be an additional early diagnostic predictor of PPOI following colorectal surgery.
\end{abstract}

\section{Introduction}

Postoperative ileus (POI) is described as a transient impairment of bowel motility that may occur following major

Correspondence to: Professor Long Cui, Department of Colorectal Surgery, Xinhua Hospital Affiliated to Shanghai Jiaotong University School of Medicine, 1665 Kongjiang Road, Shanghai 200092, P.R. China

E-mail: longcuidr@yahoo.com.cn

*Contributed equally

Key words: prolonged postoperative ileus, colorectal surgery, peritoneal cytokines, diagnostic predictor surgery (1). The process of postoperative bowel recovery following abdominal surgery, including colectomy, typically lasts for 3-5 days (2). Prolonged postoperative ileus (PPOI) usually manifests with nausea, vomiting, abdominal pain and distension and/or delay in the passage of flatus and stool. PPOI is associated with prolonged hospitalization and readmission and high morbidity and mortality (3). Although PPOI is a severe condition that requires immediate diagnosis and appropriate treatment (4), the accurate and early diagnosis of PPOI remains difficult due to a lack of appropriate laboratory parameters (5). Early detection of PPOI is critical for early intervention and minimizing mortality.

Cytokines are pleiotropic substances with a diversity of functions (6) and the cytokines IL-1 $\beta$, IL- 6 and TNF- $\alpha$ are significant mediators of the acute-phase response in humans $(6,7)$. It has been previously reported that peritoneal cytokines are sensitive indicators of the postoperative inflammatory reaction and may be predictors of serious surgical complications (8). In addition, monitoring peritoneal cytokine levels following elective gastrointestinal surgery may indicate severe intra-abdominal complications (9) and the present study investigated whether the measurement of peritoneal cytokines was an early predictor of PPOI following colorectal surgery.

\section{Patients and methods}

Patients. In the present study, 100 consecutive patients (60 male and 40 female) with carcinoma of the sigmoid or rectum and scheduled to undergo elective open anterior resection under general anesthesia between January and December 2011 were included. Of note, ileostomy was not perfomed on patients during surgery. Postoperative care for all patients included an analgesia protocol using a patient-controlled analgesia (PCA) pump of morphine chlorhydrate for $48 \mathrm{~h}$ and the dose of morphine chlorhydrate used was recorded. Exclusion criteria included patients who had received preoperative radiotherapy or chemotherapy, undergone emergency surgery and received anti-inflammatory drugs including corticosteroids and immunosuppressants preoperatively for infectious or inflammatory diseases. The mean age of the 100 eligible patients was 59 years (range, 24-87 years) at the time of surgery. The site of the carcinoma was the sigmoid colon in 26 patients and the rectum 
Table I. Data of patients receiving anterior resection of the sigmoid or rectum.

\begin{tabular}{lccr}
\hline Variables & No-PPOI & PPOI & P-value \\
\hline Age, years & $61.6 \pm 17.6$ & $53.37 \pm 8.41$ & 0.192 \\
Gender & 54 & 6 & 0.471 \\
Male & 38 & 2 & \\
Female & $23.62 \pm 4.62$ & $25.87 \pm 4.48$ & 0.213 \\
BMI & & & 0.160 \\
Site of carcinoma & 24 & 2 & \\
Sigmoid colon & 68 & 6 & 0.315 \\
Rectum & $186.32 \pm 12.17$ & $184.43 \pm 32.60$ & 0.447 \\
Blood loss, ml & $164.68 \pm 30.62$ & $194.84 \pm 127.30$ & 0.852 \\
Operative time, min & $188.22 \pm 117.61$ & $10.00 \pm 1.41$ & 0.001 \\
Dose of morphine during PCA, mg & $3.09 \pm 1.09$ & $35.43 \pm 12.21$ & 0.043 \\
Time to first passage of flatus, days & $15.62 \pm 4.46$ & & \\
Postoperative hospital stay, days &
\end{tabular}

PCA, patient-controlled analgesia; PPOI, prolonged postoperative ileus. Data are presented as the mean \pm SD.

in 74 patients. All the patients underwent bowel resection with end-to-end anastomosis with the aid of circular staplers. Prior to the closure of the peritoneum, a silastic drainage tube was placed in the pelvic base of all the patients and removed 5-12 days following surgery. This study was approved by the ethics committee of Xinhua Hospital Affiliated to Shanghai Jiaotong University School of Medicine. Written informed consent was obtained from the patients.

Definition of PPOI. POI is commonly observed $~ 3-5$ days following major abdominal surgery and presents a number of clinical symptoms, including abdominal pain or distension, nausea or vomiting, the inhibited passage of feces and/or flatus and abnormal bowel sounds. POI develops in the majority of patients undergoing abdominal surgery and it is considered to be a normal phenomenon during the postoperative course. However, a previous study (1) reported that among patients who underwent abdominal surgery, $7 \%$ developed PPOI with the observation of clinical manifestations and radiological imaging for ileus, which usually required clinical intervention. In addition, findings of another study demonstrated that the recovery of small bowel motility and absorption occurs within hours of surgery, whereas gastric and colonic functions may require 2-5 days (10). An improved clinical definition of PPOI is an ileus that persists for $>6$ days following surgery with evident clinical symptoms and radiological imaging which must be diagnosed at an early stage for immediate treatment (11). This definition was utilized in the present study and therefore, patients were divided into two groups: PPOI (with evident clinical symptoms and radiological imaging of PPOI) and No-PPOI (with no clinical symptoms and radiological imaging of PPOI).

Sampling. Samples of abdominal exudate were collected via abdominal drains on postoperative days 1, 3 and 5 for detection. Samples were obtained as fresh material within $2 \mathrm{~h}$ of the morning emptying of the drainage bag. Measurements of $5 \mathrm{ml}$ abdominal exudate were collected into a vacutainer tube and immediately centrifuged at $1,400 \mathrm{x} g$ for $15 \mathrm{~min}$. Samples were stored at $-80^{\circ} \mathrm{C}$ until analysis. The levels of IL-1 $\beta$, IL-6 and TNF- $\alpha$ were measured by enzyme-linked immunosorbent assays (R\&D Systems, Minneapolis, MN, USA). Each assay was performed in duplicate and a standard curve was constructed using recombinant cytokines. The minimum sensitivity of each cytokine assay was $2.0 \mathrm{pg} / \mathrm{ml}$.

Statistical analysis. Comparison of frequencies between the groups was analysed using the $\chi^{2}$ test with Yates' correction. Continuous data were presented as the mean \pm SD. Mean values between the two groups were compared using the unpaired t-test. For comparisons involving more than two groups, a one-way analysis of variance was applied and changes with time were analysed using the paired t-test. $\mathrm{P}<0.05$ was considered to indicate a statistically significant difference.

\section{Results}

Clinical characteristics. PPOI occurrence was clinically proven in $8 / 100$ patients $(8 \%$; 6 male and 2 female) who underwent elective open resection for carcinoma of the sigmoid or rectum. The median number of days to PPOI diagnosis was 12 days (range, 10-15 days) following surgery. Conservative treatment was administered to 6/8 PPOI patients to promote bowel movement and the remaining 2 patients were urgently reoperated on for adhesiolysis. All 8 PPOI patients recovered well following treatment and there were no mortalities for the duration of the present study. No significant differences were observed among the majority of clinical variables, including age, gender, BMI, location of carcinoma, intraoperative blood loss, operative time, dose of morphine during PCA and duration of postoperative hospitalization in the No-PPOI group when compared with that of the PPOI group. However, the number of days to the first 
Table II. Peritoneal cytokine levels on postoperative days 1, 3 and 5.

\begin{tabular}{lccc}
\hline Day & $\begin{array}{c}\mathrm{IL}-1 \beta \\
(\mathrm{pg} / \mathrm{ml})\end{array}$ & $\begin{array}{c}\mathrm{IL}-6 \\
(\mathrm{pg} / \mathrm{ml})\end{array}$ & $\begin{array}{c}\text { TNF- } \alpha \\
(\mathrm{pg} / \mathrm{ml})\end{array}$ \\
\hline 1 & $152 \pm 5.4$ & $52,000 \pm 2600$ & $312 \pm 10.7$ \\
3 & $126 \pm 4.8$ & $40,000 \pm 2500$ & $218 \pm 8.9$ \\
5 & $86 \pm 5.1$ & $36,700 \pm 2100$ & $197 \pm 9.2$
\end{tabular}

IL, interleukin; TNF, tumour necrosis factor. Data are presented as the mean $\pm \mathrm{SD}$.

passage of flatus was significantly higher in the PPOI group when compared with that of the No-PPOI group (Table I).

Peritoneal cytokines. The values of IL-1 $\beta$, IL-6 and TNF- $\alpha$ levels on postoperative days 1, 3 and 5 are shown in Table II. The impact between patient demographic factors on day 1 and peritoneal cytokine production was also examined (Table III). Age $(<60 / \geq 60$ years), gender, normal or poor $(>10 \%$ loss of body weight) preoperative nutritional status, site of carcinoma (sigmoid colon/rectum) and the Dukes' stage $(\mathrm{A} / \mathrm{B} / \mathrm{C})$ did not affect the peritoneal cytokine levels. The changes in peritoneal cytokine level for a 5-day duration following surgery are shown in Fig. 1. No significant differences were noted on postoperative days 1 and 3 among the levels of IL- $1 \beta$, IL- 6 and TNF- $\alpha$ and the 8 patients who developed PPOI when compared with those of the 92 patients that did not develop PPOI. However, on postoperative day 5 the levels of IL-1 $\beta$, IL- 6 and TNF- $\alpha$ were significantly higher in patients who developed PPOI when compared with those of patients who did not develop PPOI. The levels of IL-1 $\beta$, IL- 6 and TNF- $\alpha$ decreased in the 92 patients without PPOI and significantly increased in the 8 patients with PPOI. During the first 5 days following surgery the IL-1 $\beta$ levels increased in $7 / 8$ patients $(88 \%)$ with PPOI vs. $16 / 92$ patients $(17 \%)$ without PPOI ( $\mathrm{P}=0.0001)$, the IL-6 levels increased in $8 / 8$ patients $(100 \%)$ with PPOI vs. $15 / 92$ patients $(16 \%)$ without PPOI $(\mathrm{P}<0.0001)$ and the TNF- $\alpha$ levels increased in $8 / 8$ patients $(100 \%)$ with PPOI vs. $13 / 92$ patients $(14 \%)$ without PPOI $(\mathrm{P}<0.0001)$.

\section{Discussion}

Acute bowel obstruction is a major cause of morbidity, increased hospitalization costs and emergency conversion surgery $(11,12)$. Diagnosis of bowel obstruction traditionally occurs via classic signs including tachycardia, hypotension, fever, constant pain, peritoneal signs, leukocytosis, base deficit and metabolic acidosis (13) and abdominal CT (14). The immediate and appropriate treatment of this condition is critical, however, there are no accurate diagnostic methods or tools currently available (15). Although the combined evaluation of clinical, laboratory and radiological observations is recommended, the preoperative early diagnosis of bowel obstructions cannot be made or excluded reliably by any identified parameters, individual or combined or even by experienced clinical judgement (16).
The aim of the present study was to evaluate the predictive value of peritoneal cytokines for the early diagnosis of bowel obstructions in patients following surgery for colorectal carcinoma. Although the value of plasma cytokines for the diagnosis of PPOI has been evaluated in previous studies (17), it has not been investigated in abdominal exudate.

Peritoneal cytokines respond extensively following abdominal surgery and therefore must be a significant marker of postoperative complications (9). The IL-1 $\beta$, IL- 6 and TNF- $\alpha$ levels were measured on postoperative days 1,3 and 5 to investigate the correlation between changes in cytokine levels and the development of PPOI. In the normal postoperative progression, the peak of peritoneal cytokine production is observed on postoperative day 1 and subsequently decreases with time (18). The increased levels of peritoneal cytokines, prior to marked clinical symptoms, must be a significant marker to predict future intraabdominal ileus.

Soybel and Zinner (19) reported that ileus is correlated with the increased expression of local inflammatory cytokines and chemokines, leukocyte infiltration into the muscularis and the release of mediators from resident and infiltrating leukocytes. These mediators are capable of directly inhibiting the contraction of intestinal smooth muscle and subsequent progression of ileus.

A systemic cytokine network has been suggested to induce impaired gastric electrical activity with ileus status (20). The local inflammatory status may have a significant modulating role and correlations between ileus and increased systemic levels of proinflammatory cytokines (IL-1 $\beta$, IL- 6 and TNF- $\alpha$ ) were also identified. Previous studies (19) have revealed that the dense network of macrophages specific to the muscularis is rapidly activated during impaired intestinal motility, thus initiating an inflammatory cascade of events, including the upregulation of cytokines, chemokines and kinetically active substances (21).This local inflammatory status results in the recruitment and extravasation of leukocytes into the circular muscle layer. In conjunction with an increased expression of adhesion molecules, leukocytes inflitrate through the muscularis externa. The secretion of various potent leukocytic products directly succeeds and inhibits the contraction of gastrointestinal smooth muscle.

At present, a marked number of clinical studies have demonstrated the significance of cytokines as an early and reliable diagnostic and prognostic tool in several conditions, including infections, infectious and non-infectious SIRS, sepsis, acute pancreatitis and other postoperative complications (22). However, compared with that of systemic (plasma) cytokines, peritoneal cytokines respond extensively following major abdominal surgery, indicating that the measurement of peritoneal cytokines is a likely method to determine postoperative inflammatory reaction (8) which may progress to POI. In future studies, the preoperative systemic, as well as postoperative systemic and peritoneal cytokine measurements are likely to be performed together to investigate whether the cytokines in the peritoneal fluid are affected by the systemic circulation. In addition, the systemic and peritoneal cytokine levels following surgery are likely to be compared to indicate which is the most suitable for determining PPOI.

Results of the present study demonstrated that the estimation of cytokine levels from abdominal exudate may prove 
Table III. Peritoneal cytokine production level on postoperative day 1 vs. patient demographic factors.

\begin{tabular}{|c|c|c|c|c|}
\hline Variables & $\mathrm{n}$ & $\begin{array}{c}\mathrm{IL}-1 \beta \\
(\mathrm{pg} / \mathrm{ml})\end{array}$ & $\begin{array}{c}\text { IL-6 } \\
(\mathrm{pg} / \mathrm{ml})\end{array}$ & $\begin{array}{l}\text { TNF- } \alpha \\
(\mathrm{pg} / \mathrm{ml})\end{array}$ \\
\hline \multicolumn{5}{|l|}{ Age, years } \\
\hline$<60$ & 54 & $149 \pm 6.4$ & $44000 \pm 2,800$ & $306 \pm 12$ \\
\hline$\geq 60$ & 46 & $157 \pm 5.6$ & $53,200 \pm 2,900$ & $316 \pm 11$ \\
\hline P-value & & 0.78 & 0.66 & 0.51 \\
\hline \multicolumn{5}{|l|}{ Gender } \\
\hline Male & 60 & $157 \pm 7.7$ & $54,200 \pm 2,600$ & $298 \pm 12$ \\
\hline Female & 40 & $149 \pm 6.8$ & $52,000 \pm 2,800$ & $325 \pm 10$ \\
\hline P-value & & 0.82 & 0.53 & 0.23 \\
\hline \multicolumn{5}{|c|}{ Preoperative nutritional status } \\
\hline Normal & 82 & $150 \pm 7.2$ & $52,400 \pm 2,500$ & $312 \pm 11$ \\
\hline Poor & 18 & $156 \pm 7.5$ & $50,800 \pm 5,100$ & $316 \pm 17$ \\
\hline P-value & & 0.47 & 0.89 & 0.72 \\
\hline \multicolumn{5}{|l|}{ Site of carcinoma } \\
\hline Sigmoid colon & 26 & $148 \pm 5.2$ & $49,800 \pm 2,800$ & $314 \pm 13$ \\
\hline Rectum & 74 & $151 \pm 6.2$ & $52,100 \pm 3,700$ & $307 \pm 12$ \\
\hline P-value & & 0.62 & 0.34 & 0.45 \\
\hline \multicolumn{5}{|l|}{ Dukes' stage } \\
\hline A & 16 & $148 \pm 8.9$ & $48,600 \pm 5,300$ & $314 \pm 19$ \\
\hline $\mathrm{B}$ & 37 & $151 \pm 7.4$ & $54,300 \pm 4,100$ & $309 \pm 13$ \\
\hline $\mathrm{C}$ & 47 & $144 \pm 6.2$ & $51,000 \pm 4,400$ & $311 \pm 15$ \\
\hline P-value & & 0.91 & 0.94 & 0.88 \\
\hline
\end{tabular}

IL, interleukin; TNF, tumour necrosis factor. Data are presented as mean $\pm \mathrm{SD}$.

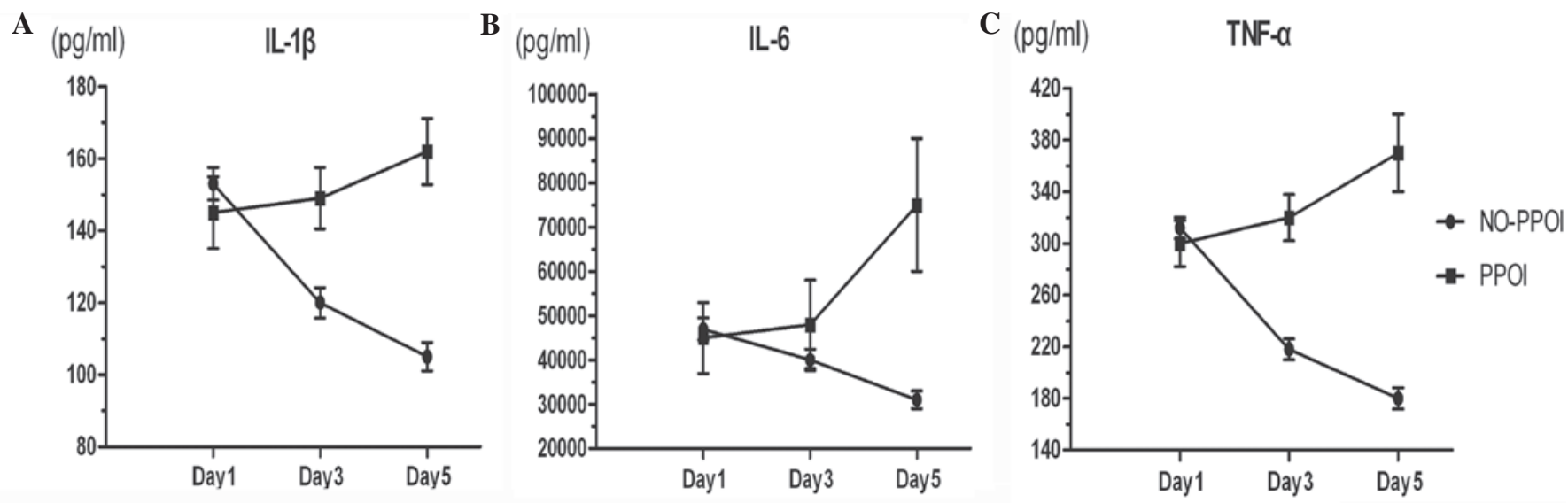

Figure 1. No significant differences were observed between the peritoneal cytokine levels on postoperative days 1 and 3 and patients who developed PPOI when compared with those that did not develop PPOI (day 1: IL-1 $\beta, P=0.23$; IL-6, $\mathrm{P}=0.35$ and TNF- $\alpha, \mathrm{P}=0.62$ and day 3: IL-1 $\beta, \mathrm{P}=0.34 ; \mathrm{IL}-6, \mathrm{P}=0.65$ and TNF- $\alpha$, $\mathrm{P}=0.29$ ). By contrast, cytokine levels on postoperative day 5 were significantly higher in patients who developed PPOI when compared with patients that did not develop PPOI (IL-1 $\beta, \mathrm{P}=0.012$; IL-6, $\mathrm{P}<0.0001$ and TNF- $\alpha, \mathrm{P}<0.0001$ ). Cytokine levels significantly increased in the first 5 days following surgery in patients who developed PPOI (IL-1 $\beta, \mathrm{P}=0.045$; IL-6, $\mathrm{P}=0.03$; TNF- $\alpha, \mathrm{P}=0.02$ ) and significantly decreased in patients who did not develop PPOI (IL-1 $\beta, \mathrm{P}<0.0001$; IL-6, P<0.0001; TNF- $\alpha, \mathrm{P}<0.0001)$. IL, interleukin; TNF, tumour necrosis factor; PPOI, prolonged postoperative ileus. Data are presented as the mean \pm SD.

to be an early diagnostic tool that is likely to support the decision-making process of surgeons for the early detection of PPOI following colorectal surgery. The measurement of peritoneal cytokines during the early postoperative period functions as a useful diagnostic marker to predict future PPOI. Therefore, it must allow for the appropriate therapeutic intervention to minimize PPOI-related morbidity, similar to that of the use of biologics to block cytokine action. 


\section{Acknowledgements}

The present study was supported by the Science and Technology Commission of Shanghai Municipality (nos. 10DJ1400504 and 09JC1410900) and the personnel training plan of Shanghai City Health Bureau.

\section{References}

1. Bauer AJ and Boeckxstaens GE: Mechanisms of postoperative ileus. Neurogastroenterol Motil 16: 54-60, 2004.

2. Iyer S, Saunders WB and Stemkowski S: Economic burden of postoperative ileus associated with colectomy in the United States. J Manag Care Pharm 15: 485-494, 2009.

3. Wolff BG, Weese JL, Ludwig KA, Delaney CP, Stamos MJ, Michelassi F, Du W and Techner L: Postoperative ileus-related morbidity profile in patients treated with alvimopan after bowel resection. J Am Coll Surg 204: 609-616, 2007.

4. Takeuchi K, Tsuzuki Y, Ando T, Sekihara M, Hara T, Yoshikawa M and Kuwano H: Clinical studies of strangulating small bowel obstruction. Am Surg 70: 40-44, 2004.

5. Firoozmand E, Fairman N, Sklar J and Waxman K: Intravenous interleukin-6 levels predict need for laparotomy in patients with bowel obstruction. Am Surg 67: 1145-1149, 2001.

6. Hesse DG, Tracey KJ, Fong Y, Manogue KR, Palladino MA Jr, Cerami A, Shires GT and Lowry SF: Cytokine appearance in human endotoxemia and primate bacteremia. Surg Gynecol Obstet 166: 147-153, 1988.

7. Castell JV, Andus T, Kunz D and Heinrich PC: Interleukin-6. The major regulator of acute-phase protein synthesis in man and rat. Ann N Y Acad Sci 557: 87-101, 1989.

8. Jansson K, Redler B, Truedsson L, Magnuson A, Matthiessen P, Andersson $\mathrm{M}$ and Norgren L: Intraperitoneal cytokine response after major surgery: higher postoperative intraperitoneal versus systemic cytokine levels suggest the gastrointestinal tract as the major source of the postoperative inflammatory reaction. Am J Surg 187: 372-377, 2004.

9. Herwig R, Glodny B, Kühle C, Schlüter B, Brinkmann OA, Strasser H, Senninger N and Winde G: Early identification of peritonitis by peritoneal cytokine measurement. Dis Colon Rectum 45: 514-521, 2002.
10. Hotokezaka M, Mentis EP, Patel SP, Combs MJ, Teates CD and Schirmer BD: Recovery of gastrointestinal tract motility and myoelectric activity change after abdominal surgery. Arch Surg 132: 410-417, 1997 .

11. Artinyan A, Nunoo-Mensah JW, Balasubramaniam S, Gauderman J, Essani R, Gonzalez-Ruiz C, Kaiser AM and Beart RW Jr: Prolonged postoperative ileus-definition, risk factors, and predictors after surgery. World J Surg 32: 1495-1500, 2008.

12. Lo OS, Law WL, Choi HK, Lee YM, Ho JW and Seto CL: Early outcomes of surgery for small bowel obstruction: analysis of risk factors. Langenbecks Arch Surg 392: 173-178, 2007.

13. Yamamoto T, Umegae S, Kitagawa T and Matsumoto K: The value of plasma cytokine measurement for the detection of strangulation in patients with bowel obstruction: a prospective, pilot study. Dis Colon Rectum 48: 1451-1459, 2005.

14. Wiesner W, Khurana B, Ji H and Ros PR: CT of acute bowel ischemia. Radiology 226: 635-650, 2003.

15. Bickell NA, Federman AD and Aufses AH Jr: Influence of time on risk of bowel resection in complete small bowel obstruction. J Am Coll Surg 201: 847-854, 2005.

16. Jeong WK, Lim SB, Choi HS and Jeong SY: Conservative management of adhesive small bowel obstructions in patients previously operated on for colorectal cancer. J Gastrointest Surg 12: 926-932, 2008.

17. Maruna P, Frasko R and Gürlich R: Plasma procalcitonin in patients with ileus. Relations to other inflammatory parameters. Physiol Res 57: 481-486, 2008.

18. Bertram P, Junge K, Schachtrupp A, Götze C, Kunz D and Schumpelick V: Peritoneal release of TNFalpha and IL-6 after elective colorectal surgery and anastomotic leakage. J Invest Surg 16: 65-69, 2003.

19. Soybel DI and Zinner MJ: Ileus and the macrophage. Ann Surg 237: 316-318, 2003.

20. Chachkhiani I, Gürlich R, Maruna P, Frasko R and Lindner J: The postoperative stress response and its reflection in cytokine network and leptin plasma levels. Physiol Res 54: 279-285, 2005.

21. Boeckxstaens GE: Neuroimmune interaction in the gut: from bench to bedside. Verh K Acad Geneeskd Belg 68: 329-355, 2006.

22. Nylen ES, O'Neill W, Jordan MH, Snider RH, Moore CF Lewis M, Silva OL and Becker KL: Serum procalcitonin as an index of inhalation injury in burns. Horm Metab Res 24: 439-443, 1992 . 